\title{
Multiplicity Results for Positive Solutions to Differential Systems of Singular Coupled Integral Boundary Value Problems
}

\author{
Yujun Cui \\ State Key Laboratory of Mining Disaster Prevention and Control Co-Founded by Shandong Province and \\ The Ministry of Science and Technology, Shandong University of Science and Technology, Qingdao 266590, China \\ Correspondence should be addressed to Yujun Cui; cyj720201@163.com
}

Received 16 April 2017; Accepted 11 May 2017; Published 5 June 2017

Academic Editor: Marco Spadini

Copyright (C) 2017 Yujun Cui. This is an open access article distributed under the Creative Commons Attribution License, which permits unrestricted use, distribution, and reproduction in any medium, provided the original work is properly cited.

By constructing a special cone and using a fixed-point theorem in cone, this paper investigates the existence of multiple solutions of coupled integral boundary value problems for a nonlinear singular differential system.

\section{Introduction}

In recent years, singular uncoupled boundary value problems to differential systems have been studied widely and there are many excellent results (see [1-18] and references therein). Naturally we hope there are the same excellent results on singular uncoupled boundary value problems to differential systems with coupled boundary conditions. Many researchers put their efforts to study the existence of solutions for differential systems with coupled boundary conditions (see [19-30] and references therein).

In [19], Asif and Khan addressed the question of the existence of coupled four-point boundary value conditions

$$
\begin{aligned}
-x^{\prime \prime}(t) & =f_{1}(t, x(t), y(t)), \quad t \in(0,1), \\
-y^{\prime \prime}(t) & =f_{2}(t, x(t), y(t)), \quad t \in(0,1), \\
x(0) & =y(0)=0, \\
x(1) & =\alpha y(\xi), \\
y(1) & =\beta x(\eta),
\end{aligned}
$$

where the parameters $\alpha, \beta, \xi$, and $\eta$ satisfy $\xi, \eta \in(0,1)$, $0<\alpha \beta \xi \eta<1$. The main tool in [19] is the Guo-Krasnosel'skii fixed-point theorem.
In [29], the authors studied the following nonlinear semipositone fractional differential equation with four-point coupled boundary value problem:

$$
\begin{aligned}
D_{0+}^{\alpha} u+\lambda f(t, u, v) & =0, \quad t \in(0,1), \lambda>0, \\
D_{0+}^{\alpha} v+\lambda g(t, u, v) & =0, \\
u^{(i)}(0) & =v^{(i)}(0)=0, \quad 0 \leq i \leq n-2, \\
u(1) & =a v(\xi), \\
v(1) & =b u(\eta),
\end{aligned}
$$

where $\lambda$ is a parameter, $a, b, \xi$, and $\eta$ satisfy $\xi, \eta \in(0,1)$, $0<a b \xi \eta<1, \alpha \in(n-1, n]$ is a real number and $n \geq 3$, and $D_{0+}^{\alpha} u$ is Riemann-Liouville's fractional derivative. The existence of positive solutions is established by using a nonlinear alternative of Leray-Schauder type and GuoKrasnosel'skiu fixed-point theorem in a cone.

In [20], Cui and Sun, using fixed-point index theory, studied the existence of positive solutions for superlinear differential system

$$
\begin{array}{ll}
-x^{\prime \prime}(t)=f_{1}(t, x(t), y(t)), & t \in(0,1), \\
-y^{\prime \prime}(t)=f_{2}(t, x(t), y(t)), & t \in(0,1),
\end{array}
$$




$$
\begin{aligned}
& x(0)=y(0)=0, \\
& x(1)=\alpha[y], \\
& y(1)=\beta[x],
\end{aligned}
$$

where $\alpha[x]$ and $\beta[x]$ are bounded linear functionals on $C[0,1]$ given by

$$
\begin{aligned}
& \alpha[x]=\int_{0}^{1} x(t) d A(t), \\
& \beta[x]=\int_{0}^{1} x(t) d B(t),
\end{aligned}
$$

involving Stieltjes integrals; in particular, $A$ and $B$ are functions of bounded variation with positive measures.

We should note that the nonlinear terms in two equations for the above problems have the same features. For instance, the nonlinear terms in two equations are both superlinear $[20,29]$ or both sublinear [19]. However, to the best of our knowledge, only a few papers discuss differential system under the case that the nonlinear terms of the system have different behaviors. Motivated by [19, 20, 29], the purpose of this paper is to establish the existence of multiple positive solutions for differential system with coupled integral boundary value problems (3) when $f_{1}$ is superlinear in $x$ and $y$ and $f_{2}$ is sublinear in $x$ and $y$. Also suppose that $f_{1}(t, x, y)$ may be singular at $t=0, t=1$ and $f_{2}(t, x, y)$ may be singular at $t=0, t=1, x=0$, and $y=0$. Our main features are threefold. Firstly, our study is on singular nonlinear differential systems with general boundary value conditions. Secondly, $f_{2}$ is allowed to be not only singular at $t=0$ and 1 but also singular at $x=0$ and $y=0$. Finally, a special cone is constructed to overcome difficulties due to singularities of nonlinear term.

In the rest of this section, let us list the following assumptions:

$\left(H_{1}\right) \kappa_{1}>0, \kappa_{2}>0$, and $\kappa>0$, where

$$
\begin{aligned}
& \kappa_{1}=\alpha[t]=\int_{0}^{1} t d A(t), \\
& \kappa_{2}=\beta[t]=\int_{0}^{1} t d B(t), \\
& \kappa=1-\kappa_{1} \kappa_{2} .
\end{aligned}
$$

$\left(H_{2}\right) f_{1} \in C\left((0,1) \times[0, \infty)^{2},[0, \infty)\right)$ satisfy

$$
0<\int_{0}^{1} s(1-s) f_{1}(s, 1,1) d s<+\infty,
$$

and there exist constants $\lambda_{1 j}, \mu_{1 j}\left(0<\lambda_{1 j} \leq \mu_{1 j}<1, j=1,2\right.$, $\left.\lambda_{11}+\lambda_{12}>1\right)$ such that, for $t \in(0,1), x, y \in(0, \infty)$,

$$
\begin{aligned}
& c^{\mu_{11}} f_{1}(t, x, y) \leq f_{1}(t, c x, y) \leq c^{\lambda_{11}} f_{1}(t, x, y), \\
& c^{\mu_{12}} f_{1}(t, x, y) \leq f_{1}(t, x, c y) \leq c^{\lambda_{12}} f_{1}(t, x, y),
\end{aligned}
$$

if $0<c \leq 1$.
$\left(H_{3}\right) f_{2} \in C\left((0,1) \times(0, \infty)^{2},[0, \infty)\right)$ and there exist constants $\lambda_{2 j}, \mu_{2 j}\left(-\infty<\lambda_{2 j} \leq \mu_{2 j}<1, j=1,2, \mu_{21}+\mu_{22}<\right.$ 1) such that, for $t \in(0,1), x, y \in(0, \infty)$,

$$
\begin{aligned}
c^{\mu_{21}} f_{2}(t, x, y) & \leq f_{2}(t, c x, y) \leq c^{\lambda_{21}} f_{2}(t, x, y), \\
c^{\mu_{22}} f_{2}(t, x, y) \leq f_{2}(t, x, c y) & \leq c^{\lambda_{22}} f_{2}(t, x, y), \\
& \text { if } 0<c \leq 1 .
\end{aligned}
$$

And they satisfy one of the following conditions:

$$
\left(H_{31}\right) \lambda_{21}>0, \lambda_{22}>0,0<\int_{0}^{1} s(1-s) f_{2}(s, 1,1) d s<+\infty,
$$
$\rho \int_{0}^{1} s(1-s) f_{1}(s, 1,1) d s+\rho \int_{0}^{1} s(1-s) f_{2}(s, 1,1) d s<1$.

$\left(H_{32}\right) \mu_{21}<0, \mu_{22}<0,0<\int_{0}^{1} s(1-s) f_{2}(s, s, s) d s<+\infty$, $\rho \int_{0}^{1} s(1-s) f_{1}(s, 1,1) d s+\rho \gamma^{\mu_{21}+\mu_{22}} \int_{0}^{1} s(1-s) f_{2}(s, s, s) d s<1$.

$\left(H_{33}\right) \lambda_{21}>0, \mu_{22}<0,0<\int_{0}^{1} s(1-s) f_{2}(s, 1, s) d s<+\infty$, $\rho \int_{0}^{1} s(1-s) f_{1}(s, 1,1) d s+\rho \gamma^{\mu_{22}} \int_{0}^{1} s(1-s) f_{2}(s, 1, s) d s<1$.

$$
\left(H_{34}\right) \mu_{21}<0, \lambda_{22}>0,0<\int_{0}^{1} s(1-s) f_{2}(s, s, 1) d s<
$$
$+\infty . \rho \int_{0}^{1} s(1-s) f_{1}(s, 1,1) d s+\rho \gamma^{\mu_{21}} \int_{0}^{1} s(1-s) f_{2}(s, s, 1) d s<1$, where

$$
\begin{aligned}
0<\gamma=\frac{\nu}{\rho}<1, \\
\rho=\max \left\{\frac{\alpha[t]}{\kappa} \beta[1]+1, \frac{\beta[t]}{\kappa} \alpha[1]+1, \frac{1}{\kappa} \beta[1], \frac{1}{\kappa}\right. \\
\quad \alpha[1]\}, \\
\nu=\min \left\{\frac{\alpha[t]}{\kappa} \beta[t(1-t)], \frac{\beta[t]}{\kappa} \alpha[t(1-t)], \frac{1}{\kappa}\right. \\
\left.\quad \cdot \beta[t(1-t)], \frac{1}{\kappa} \alpha[t(1-t)]\right\} .
\end{aligned}
$$

Remark 1. Equations (7)-(8) imply

$$
\begin{aligned}
c^{\lambda_{i 1}} f_{i}(t, x, y) \leq f_{i}(t, c x, y) \leq c^{\mu_{i 1}} f_{i}(t, x, y) & \\
c^{\lambda_{i 2}} f_{i}(t, x, y) \leq f_{i}(t, x, c y) \leq c^{\mu_{i 2}} f_{i}(t, x, y) & \\
& \text { if } c \geq 1, i=1,2 .
\end{aligned}
$$

Conversely, (11) implies (7)-(8).

Remark 2. Equation (7) implies

$$
\begin{aligned}
& f_{1}\left(t, x_{1}, y_{1}\right) \leq f_{1}\left(t, x_{2}, y_{2}\right) \\
& \quad \text { if } 0<x_{1} \leq x_{2}, 0<y_{1} \leq y_{2} .
\end{aligned}
$$

Remark 3. $\left(H_{31}\right)$ implies that $f_{2}(t, x, y)$ is nondecreasing in $x$ and $y$.

$\left(H_{32}\right)$ implies that $f_{2}(t, x, y)$ is nonincreasing in $x$ and $y$.

$\left(H_{33}\right)$ implies that $f_{2}(t, x, y)$ is nondecreasing in $x$ and nonincreasing in $y$.

$\left(H_{34}\right)$ implies that $f_{2}(t, x, y)$ is nonincreasing in $x$ and nondecreasing in $y$. 


\section{Main Results}

For each $u \in E:=C[0,1]$, we write $\|u\|=\max \{|u(t)|: t \in$ $[0,1]\}$. Clearly, $(E,\|\cdot\|)$ is a Banach space. Similarly, for each $(x, y) \in E \times E$, we write $\|(x, y)\|_{1}=\max \{\|x\|,\|y\|\}$. For any real constant $r>0$, define $\Omega_{r}=\left\{(x, y) \in E \times E:\|(x, y)\|_{1}<r\right\}$. Define

$$
\begin{aligned}
P & =\left\{(x, y) \in E \times E: x(t) \geq \gamma t\|(x, y)\|_{1}, y(t)\right. \\
& \left.\geq \gamma t\|(x, y)\|_{1}, t \in[0,1]\right\},
\end{aligned}
$$

where $\gamma$ is given by $(9)$. Clearly, $\left(E \times E,\|\cdot\|_{1}\right)$ is a Banach space and $P$ is a cone of $E \times E$.

Remark 4 . The cone $P$ defined by (13) is completely different from the cone used in the uncoupled boundary value problems. This means that the cone $P$ has the following property:

$$
\begin{array}{cl}
\text { if } \quad & (x, y) \in P \backslash\{\theta\}, \\
\text { then } \quad & \gamma t\|(x, y)\|_{1} \leq x(t), \\
& y(t) \leq\|(x, y)\|_{1},
\end{array}
$$

$$
\text { for } t \in[0,1] \text {, }
$$

which is crucial in the definition of $T$ and in the proof of Lemma 9.

Our main result is the following theorems.

Theorem 5. Assume that $\left(H_{1}\right),\left(H_{2}\right)$, and $\left(H_{3}\right)$ are satisfied. Then differential system (3) has at least two positive solutions $\left(x_{1}, y_{1}\right),\left(x_{2}, y_{2}\right) \in C[0,1] \times C[0,1]$ such that $0<\left\|\left(x_{1}, y_{1}\right)\right\|_{1}<$ $1<\left\|\left(x_{2}, y_{2}\right)\right\|_{1}$.

Note. We need only to prove this theorem under condition $\left(H_{33}\right)$, since the proof is similar when $\left(H_{31}\right)$ or $\left(H_{32}\right)$ or $\left(H_{34}\right)$ is satisfied.

The proof of Theorem 5 is based on the following theorem in [31].

Lemma 6. Let $E$ be a Banach space and $P$ a cone in E. Suppose that $\Omega_{1}$ and $\Omega_{2}$ are two bounded open subsets of $E$ with $\theta \in \Omega_{1}$, $\overline{\Omega_{1}} \subset \Omega_{2}$. Let operator $T: P \cap\left(\overline{\Omega_{2}} \backslash \Omega_{1}\right) \rightarrow P$ be completely continuous. Suppose that one of the two conditions

$$
\begin{aligned}
& \text { (i) } \quad\|T x\| \leq\|x\|, \forall x \in P \cap \partial \Omega_{1}, \\
&\|T x\| \geq\|x\|, \quad \forall x \in P \cap \partial \Omega_{2}, \\
& \text { (ii) } \quad\|T x\| \geq\|x\|, \quad \forall x \in P \cap \partial \Omega_{1}, \\
& \quad\|T x\| \leq\|x\|, \quad \forall x \in P \cap \partial \Omega_{2}
\end{aligned}
$$

Lemma 7 (see [20]). Assume that $\left(H_{1}\right)$ holds. Let $u, v \in E$; then the system of BVPs

$$
\begin{aligned}
& -x^{\prime \prime}(t)=u(t), \\
& -y^{\prime \prime}(t)=v(t),
\end{aligned}
$$

$$
t \in[0,1]
$$

$$
\begin{aligned}
& x(0)=y(0)=0, \\
& x(1)=\alpha[y], \\
& y(1)=\beta[x]
\end{aligned}
$$

has integral representation

$$
\begin{aligned}
& x(t)=\int_{0}^{1} G_{1}(t, s) u(s) d s+\int_{0}^{1} H_{1}(t, s) v(s) d s, \\
& y(t)=\int_{0}^{1} G_{2}(t, s) v(s) d s+\int_{0}^{1} H_{2}(t, s) u(s) d s,
\end{aligned}
$$

where

$$
\begin{gathered}
k(t, s)= \begin{cases}t(1-s), & 0 \leq t \leq s \leq 1, \\
s(1-t), & 0 \leq s \leq t \leq 1,\end{cases} \\
G_{1}(t, s)=\frac{k_{1} t}{\kappa} \int_{0}^{1} k(s, \tau) d B(\tau)+k(t, s), \\
H_{1}(t, s)=\frac{t}{\kappa} \int_{0}^{1} k(s, \tau) d A(\tau), \\
G_{2}(t, s)=\frac{k_{2} t}{\kappa} \int_{0}^{1} k(s, \tau) d A(\tau)+k(t, s), \\
H_{2}(t, s)=\frac{t}{\kappa} \int_{0}^{1} k(s, \tau) d B(\tau) .
\end{gathered}
$$

Remark 8 (see [20]). From (13) and $\left(H_{1}\right)$, for $t \in[0,1]$, we have

$$
\begin{aligned}
& G_{i}(t, s) \leq \rho s(1-s), \\
& H_{i}(t, s) \leq \rho s(1-s), \\
& G_{i}(t, s) \geq v t s(1-s), \\
& H_{i}(t, s) \geq v t s(1-s), \\
& i=1,2 .
\end{aligned}
$$

Define an operator $T: P \backslash\{\theta\} \rightarrow P$ by

$$
T(x, y)=\left(T_{1}(x, y), T_{2}(x, y)\right),
$$

where operators $T_{1}, T_{2}: P \backslash\{\theta\} \rightarrow Q=\{u \in E \mid u(t) \geq 0, t \in$ $[0,1]\}$ are defined by

$$
\begin{aligned}
T_{1}(x, y)(t)= & \int_{0}^{1} G_{1}(t, s) f_{1}(s, x(s), y(s)) d s \\
& +\int_{0}^{1} H_{1}(t, s) f_{2}(s, x(s), y(s)) d s
\end{aligned}
$$




$$
\begin{array}{cl}
T_{2}(x, y)(t)=\int_{0}^{1} G_{2}(t, s) f_{2}(s, x(s), y(s)) d s & G_{1}(t, s) \geq \gamma t H_{2}(\tau, s), \\
+\int_{0}^{1} H_{2}(t, s) f_{1}(s, x(s), y(s)) d s, & H_{2}(t, s) \geq \gamma t G_{1}(\tau, s), \\
& t \in[0,1] .
\end{array}
$$

(21) Hence, for $(x, y) \in P \cap\left(\overline{\Omega_{b}} \backslash \Omega_{a}\right), t \in[0,1]$, we have

Lemma 9. Assume that $\left(H_{1}\right),\left(H_{2}\right)$, and $\left(H_{3}\right)$ hold. Then, for any $0<a<b<+\infty, T: P \cap\left(\overline{\Omega_{b}} \backslash \Omega_{a}\right) \rightarrow P$ is a completely continuous operator.

Proof. For $(x, y) \in P \cap\left(\overline{\Omega_{b}} \backslash \Omega_{a}\right)$, let $c$ be a positive number such that $\|(x, y)\|_{1} / c<1$ and $c>1$. From $\left(H_{2}\right),\left(H_{3}\right)$, and Remark 2, we have

$$
\begin{aligned}
f_{1}(t, x(t), y(t)) & \leq f_{1}(t, c, c) \leq c^{\mu_{11}+\mu_{12}} f_{1}(t, 1,1), \\
f_{2}(t, x(t), y(t)) & \leq f_{2}\left(t, c, \gamma\|(x, y)\|_{1} t\right) \\
& \leq c^{\mu_{21}} f_{2}\left(t, 1, \min \left\{\gamma\|(x, y)\|_{1}, 1\right\} t\right) \\
& \leq c^{\mu_{21}} \min \{\gamma a, 1\}^{\lambda_{22}} f_{2}(t, 1, t) .
\end{aligned}
$$

Hence for any $t \in[0,1]$, by Remark 8 , we get

$$
\begin{aligned}
& T_{i}(x, y)(t) \\
& \leq \rho \int_{0}^{1} s(1-s) f_{1}(s, x(s), y(s)) d s \\
& \quad+\rho \int_{0}^{1} s(1-s) f_{2}(s, x(s), y(s)) d s \\
& \leq \rho \int_{0}^{1} s(1-s) f_{1}(s, c, c) d s \\
& \quad+\rho \int_{0}^{1} s(1-s) f_{2}\left(s, c, \gamma s\|(x, y)\|_{1}\right) d s \\
& \leq \rho c^{\mu_{11}+\mu_{12}} \int_{0}^{1} s(1-s) f_{1}(s, 1,1) d s \\
& \quad+\rho c^{\mu_{21}} \min \{\gamma a, 1\}^{\lambda_{22}} \int_{0}^{1} s(1-s) f_{2}(s, 1, s) d s
\end{aligned}
$$

Thus, $T$ is well defined on $P \cap\left(\overline{\Omega_{b}} \backslash \Omega_{a}\right)$.

Next we show that $T\left(P \cap\left(\overline{\Omega_{b}} \backslash \Omega_{a}\right)\right) \subset P$. By Remark 8, for $\tau, t, s \in[0,1]$, we obtain

$$
\begin{aligned}
& G_{i}(t, s) \geq \gamma t G_{i}(\tau, s), \\
& H_{i}(t, s) \geq \gamma t H_{i}(\tau, s), \\
& \qquad i=1,2, \\
& H_{1}(t, s) \geq \gamma t G_{2}(\tau, s),
\end{aligned}
$$

$$
\begin{aligned}
T_{1}(x, y)(t)= & \int_{0}^{1} G_{1}(t, s) f_{1}(s, x(s), y(s)) d s \\
& +\int_{0}^{1} H_{1}(t, s) f_{2}(s, x(s), y(s)) d s \\
\geq & \gamma t \int_{0}^{1} G_{1}(\tau, s) f_{1}(s, x(s), y(s)) d s \\
& +\gamma t \int_{0}^{1} H_{1}(\tau, s) f_{2}(s, x(s), y(s)) d s \\
= & \gamma t T_{1}(x, y)(\tau), \\
T_{1}(x, y)(t)= & \int_{0}^{1} G_{1}(t, s) f_{1}(s, x(s), y(s)) d s \\
& +\int_{0}^{1} H_{1}(t, s) f_{2}(s, x(s), y(s)) d s \\
\geq & \gamma t \int_{0}^{1} H_{2}(\tau, s) f_{1}(s, x(s), y(s)) d s \\
& +\gamma t \int_{0}^{1} G_{2}(\tau, s) f_{2}(s, x(s), y(s)) d s \\
= & \gamma t T_{2}(x, y)(\tau) .
\end{aligned}
$$

Then $T_{1}(x, y)(t) \geq \gamma t\left\|T_{1}(x, y)\right\|$ and $T_{1}(x, y)(t) \geq \gamma t \| T_{2}(x$, $y) \|$, that is, $T_{1}(x, y)(t) \geq \gamma t\left\|\left(T_{1}(x, y), T_{2}(x, y)\right)\right\|_{1}$. In the same way, we can prove that $T_{2}(x, y)(t) \geq \gamma t \|\left(T_{1}(x, y)\right.$, $\left.T_{2}(x, y)\right) \|_{1}$. Therefore, $T\left(P \cap\left(\overline{\Omega_{b}} \backslash \Omega_{a}\right)\right) \subset P$.

Moreover, $T: P \cap\left(\overline{\Omega_{b}} \backslash \Omega_{a}\right) \rightarrow P$ is a completely continuous operator. This is a standard textbook result using Ascoli-Arzela theorem (see, e.g., [31]) and is omitted.

Proof of Theorem 5. By $\left(\mathrm{H}_{2}\right)$ and $\left(\mathrm{H}_{3}\right)$, we can get

$$
\begin{aligned}
& t^{\mu_{11}+\mu_{12}} f_{1}(t, 1,1) \leq f_{1}(t, t, t) \leq f_{1}(t, 1,1) \\
& t^{\mu_{21}-\lambda_{22}} f_{2}(t, 1, t) \leq f_{2}(t, t, 1) \leq f_{2}(t, 1, t)
\end{aligned}
$$

$$
t \in(0,1) .
$$

This implies that

$$
\begin{aligned}
& 0<\int_{0}^{1} s(1-s) f_{1}(s, s, s) d s<+\infty \\
& 0<\int_{0}^{1} s(1-s) f_{2}(s, s, 1) d s<+\infty .
\end{aligned}
$$


Choose constants $r$ and $R$ such that

$$
\begin{aligned}
& R>\max \left\{\gamma^{-1}, 1,\left(\frac{\nu}{4}\right.\right. \\
& \left.\left.\cdot \gamma^{\lambda_{11}+\lambda_{12}} \int_{0}^{1} s(1-s) f_{1}(s, s, s) d s\right)^{-1 /\left(\lambda_{11}+\lambda_{12}-1\right)}\right\}, \\
& 0<r<\min \left\{\frac{1}{2},\left(\frac{\nu}{4}\right.\right. \\
& \left.\left.\cdot \gamma^{\mu_{21}} \int_{0}^{1} s(1-s) f_{2}(s, s, 1) d s\right)^{1 /\left(1-\mu_{21}-\mu_{22}\right)}\right\} .
\end{aligned}
$$

It follows from Lemma 9 that $T: P \cap\left(\overline{\Omega_{R}} \backslash \Omega_{r}\right) \rightarrow P$ is a completely continuous operator. Moreover, by Lemma 7 , if $(x, y) \in P \cap\left(\overline{\Omega_{R}} \backslash \Omega_{r}\right)$ is a fixed point of $T$, then $(x, y)$ is a solution of differential system (3).

For any $t \in[0,1],(x, y) \in P \cap \partial \Omega_{r}$, it follows from the definition of cone $P$ that

$$
\gamma r t=\gamma t\|(x, y)\|_{1} \leq x(t), \quad y(t) \leq r<1 .
$$

Thus for any $(x, y) \in P \cap \partial \Omega_{r}$, by $\left(H_{33}\right)$ and Remarks 2 and 8 , we have

$$
\begin{aligned}
T_{i}(x, y)(t) & \geq \frac{\nu}{4} \int_{0}^{1} s(1-s) f_{2}(s, \gamma r s, r) d s \\
& \geq \frac{v}{4} \gamma^{\mu_{21}} r^{\mu_{21}+\mu_{22}} \int_{0}^{1} s(1-s) f_{2}(s, s, 1) d s \\
& \geq r=\|(x, y)\|_{1}, \quad i=1,2, t \in\left[\frac{1}{4}, 1\right] .
\end{aligned}
$$

Consequently,

$$
\|T(x, y)\|_{1} \geq\|(x, y)\|_{1}, \quad \forall(x, y) \in P \cap \partial \Omega_{r} .
$$

Again, for any $t \in[0,1],(x, y) \in P \cap \partial \Omega_{R}$, we have

$$
\begin{aligned}
& \gamma R t=\gamma t\|(x, y)\|_{1} \leq x(t), \\
& y(t) \leq R .
\end{aligned}
$$

Thus for any $(x, y) \in P \cap \partial \Omega_{R}$, noting that $\gamma R>1$, we have

$$
\begin{aligned}
T_{i}(x, y)(t) & \geq \frac{v}{4} \int_{0}^{1} s(1-s) f_{1}(s, \gamma R s, \gamma R s) d s \\
& \geq \frac{v}{4}(\gamma R)^{\lambda_{11}+\lambda_{12}} \int_{0}^{1} s(1-s) f_{1}(s, s, s) d s \\
& \geq R=\|(x, y)\|_{1}, \quad i=1,2, t \in\left[\frac{1}{4}, 1\right] .
\end{aligned}
$$

This guarantees

$$
\|T(x, y)\|_{1} \geq\|(x, y)\|_{1}, \quad \forall(x, y) \in P \cap \partial \Omega_{R} .
$$

On the other hand, for any $t \in[0,1],(x, y) \in P \cap \partial \Omega_{1}$, it follows from the definition of cone $P$ that

$$
\begin{gathered}
\gamma t \leq x(t), \\
y(t) \leq 1 .
\end{gathered}
$$

Thus for any $(x, y) \in P \cap \partial \Omega_{1}$, by $\left(H_{33}\right)$ and Remarks 2 and 8 , we have

$$
\begin{aligned}
T_{i}(x, y)(t) \leq & \rho \int_{0}^{1} s(1-s) f_{1}(s, 1,1) d s \\
& +\rho \int_{0}^{1} s(1-s) f_{2}(s, 1, \gamma s) d s \\
\leq & \rho \int_{0}^{1} s(1-s) f_{1}(s, 1,1) d s \\
& +\rho \gamma^{\mu_{22}} \int_{0}^{1} s(1-s) f_{2}(s, 1, s) d s<1 \\
= & \|(x, y)\|_{1}, \quad i=1,2 .
\end{aligned}
$$

That is,

$$
\|T(x, y)\|_{1}<\|(x, y)\|_{1}, \quad \forall(x, y) \in P \cap \partial \Omega_{1} .
$$

Therefore, from (31), (37), and Lemma 6, it follows that differential system (3) has one positive solution $\left(x_{1}, y_{1}\right) \in P$ with $r \leq\left\|\left(x_{1}, y_{1}\right)\right\|_{1}<1$. In the same way, from (34), (37), and Lemma 6, it follows that differential system (3) has one positive solution $\left(x_{2}, y_{2}\right) \in P$ with $1<\left\|\left(x_{1}, y_{1}\right)\right\|_{1} \leq R$.

\section{An Example}

In this section we give an example to illustrate the usefulness of our main results. Let us consider the singular differential system with coupled boundary value problem

$$
\begin{aligned}
& -x^{\prime \prime}=\frac{x^{p_{1}} y^{q_{1}}}{\pi \sqrt{t(1-t)}}, \\
& -y^{\prime \prime}=\frac{x^{p_{2}}}{4 \sqrt{y}} t, \\
& x(0)=y(0)=0, \\
& x(1)=y\left(\frac{1}{4}\right)+y\left(\frac{1}{2}\right), \\
& y(1)=\int_{0}^{1} x(t) d t,
\end{aligned}
$$

where $0 \leq p_{1}, q_{1}, p_{2}<+\infty, p_{1}+q_{1}>1, p_{2}<1$. 
Let

$$
\begin{aligned}
& A(t)= \begin{cases}0, & t \in\left[0, \frac{1}{4}\right), \\
1, & t \in\left[\frac{1}{4}, \frac{1}{2}\right), \\
2, & t \in\left[\frac{1}{2}, 1\right],\end{cases} \\
& B(t)=t \text {, } \\
& \lambda_{11}=\mu_{11}=p_{1}, \\
& \lambda_{12}=\mu_{12}=q_{1} \text {, } \\
& \lambda_{21}=\mu_{21}=p_{2} \text {, } \\
& \lambda_{22}=\mu_{22}=-\frac{1}{2} \text {, } \\
& f_{1}(t, x, y)=\frac{x^{p_{1}} y^{q_{1}}}{\pi \sqrt{t(1-t)}}, \\
& f_{2}(t, x, y)=\frac{x^{p_{2}}}{4 \sqrt{y}} t
\end{aligned}
$$

then

$$
\begin{aligned}
& \kappa_{1}=\frac{3}{4} \\
& \kappa_{2}=\frac{1}{2} \\
& \kappa=1-\kappa_{1} \kappa_{2}=\frac{5}{8}, \\
& \rho=\frac{16}{5} \\
& v=\frac{1}{5}, \\
& \gamma=\frac{1}{16} \\
& \rho \int_{0}^{1} s(1-s) f_{1}(s, 1,1) d s \\
& +\rho \gamma^{\mu_{22}} \int_{0}^{1} s(1-s) f_{2}(s, 1, s) d s=\frac{16}{5}\left(\frac{1}{8}+\frac{4}{35}\right) \\
& =\frac{134}{175}<1 \text {. }
\end{aligned}
$$

So all conditions of Theorem 5 are satisfied for (38), and our conclusion follows from Theorem 5 .

\section{Conflicts of Interest}

The author declares that they have no conflicts of interest.

\section{Acknowledgments}

This project is supported by NNSF of China (11371221, 11571207) and the Tai'shan Scholar Engineering Construction Fund of Shandong Province of China.

\section{References}

[1] R. P. Agarwal and D. O’Regan, "A coupled system of boundary value problems," Applicable Analysis. An International Journal, vol. 69, no. 3-4, pp. 381-385, 1998.

[2] R. P. Agarwal and D. O'Regan, "Multiple solutions for a coupled system of boundary value problems," Dynamics of Continuous, Discrete and Impulsive Systems. An International Journal for Theory and Applications, vol. 7, no. 1, pp. 97-106, 2000.

[3] N. A. Asif and R. A. Khan, "Multiplicity results for positive solutions of a coupled system of singular boundary value problems," Communications on Applied Nonlinear Analysis, vol. 17, no. 2, pp. 53-68, 2010.

[4] N. A. Asif, R. A. Khan, and J. Henderson, "Existence of positive solutions to a system of singular boundary value problems," Dynamic Systems and Applications, vol. 19, no. 2, pp. 395-404, 2010.

[5] Z. Bai and Y. Zhang, "The existence of solutions for a fractional multi-point boundary value problem," Computers \& Mathematics with Applications. An International Journal, vol. 60, no. 8, pp. 2364-2372, 2010.

[6] Z. Bai and Y. Zhang, "Solvability of fractional three-point boundary value problems with nonlinear growth," Applied Mathematics and Computation, vol. 218, no. 5, pp. 1719-1725, 2011.

[7] X. Cheng and C. Zhong, "Existence of positive solutions for a second-order ordinary differential system," Journal of Mathematical Analysis and Applications, vol. 312, no. 1, pp. 1423, 2005.

[8] A. M. Fink and J. A. Gatica, "Positive solutions of second order systems of boundary value problems," Journal of Mathematical Analysis and Applications, vol. 180, no. 1, pp. 93-108, 1993.

[9] J. Henderson and R. Luca, "Positive solutions for a system of second-order multi-point boundary value problems," Applied Mathematics and Computation, vol. 218, no. 10, pp. 6083-6094, 2012.

[10] G. Infante and P. Pietramala, "Existence and multiplicity of non-negative solutions for systems of perturbed Hammerstein integral equations," Nonlinear Analysis. Theory, Methods \& Applications. An International Multidisciplinary Journal, vol. 71, no. 3-4, pp. 1301-1310, 2009.

[11] G. Infante and P. Pietramala, "Eigenvalues and nonnegative solutions of a system with nonlocal BCs," Nonlinear Studies, vol. 16, no. 2, pp. 187-196, 2009.

[12] K. Q. Lan and W. Lin, "Multiple positive solutions of systems of Hammerstein integral equations with applications to fractional differential equations," Journal of the London Mathematical Society. Second Series, vol. 83, no. 2, pp. 449-469, 2011.

[13] Y. Liu and B. Yan, "Multiple solutions of singular boundary value problems for differential systems," Journal of Mathematical Analysis and Applications, vol. 287, no. 2, pp. 540-556, 2003.

[14] H. Lu, H. Yu, and Y. Liu, "Positive solutions for singular boundary value problems of a coupled system of differential equations," Journal of Mathematical Analysis and Applications, vol. 302, no. 1, pp. 14-29, 2005. 
[15] L. Liu, F. Sun, X. Zhang, and Y. Wu, "Bifurcation analysis for a singular differential system with two parameters via to topological degree theory," Lithuanian Association of Nonlinear Analysts (LANA). Nonlinear Analysis. Modelling and Control, vol. 22, no. 1, pp. 31-50, 2017.

[16] F. Sun, L. Liu, X. Zhang, and Y. Wu, "Spectral analysis for a singular differential system with integral boundary conditions," Mediterranean Journal of Mathematics, vol. 13, no. 6, pp. 47634782, 2016.

[17] Y. Wang, L. Liu, X. Zhang, and Y. Wu, "Positive solutions of an abstract fractional semipositone differential system model for bioprocesses of HIV infection," Applied Mathematics and Computation, vol. 258, pp. 312-324, 2015.

[18] Y. Wang, L. Liu, and Y. Wu, "Positive solutions for a class of higher-order singular semipositone fractional differential systems with coupled integral boundary conditions and parameters," Advances in Difference Equations, 2014:268, 24 pages, 2014.

[19] N. A. Asif and R. A. Khan, "Positive solutions to singular system with four-point coupled boundary conditions," Journal of Mathematical Analysis and Applications, vol. 386, no. 2, pp. 848-861, 2012.

[20] Y. Cui and J. Sun, "On existence of positive solutions of coupled integral boundary value problems for a nonlinear singular superlinear differential system," Electronic Journal of Qualitative Theory of Differential Equations, no. 41, 13 pages, 2012.

[21] Y. Cui and Y. Zou, "An existence and uniqueness theorem for a second order nonlinear system with coupled integral boundary value conditions," Applied Mathematics and Computation, vol. 256, pp. 438-444, 2015.

[22] Y. Cui, "Existence of solutions for coupled integral boundary value problem at resonance," Publicationes Mathematicae Debrecen, vol. 89, no. 1-2, pp. 73-88, 2016.

[23] Y. Cui, L. Liu, and X. Zhang, "Uniqueness and existence of positive solutions for singular differential systems with coupled integral boundary value problems," Abstract and Applied Analysis, vol. 2013, Article ID 340487, 2013.

[24] Y. Cui and Y. Zou, "Monotone iterative method for differential systems with coupled integral boundary value problems," Boundary Value Problems, vol. 2013, no. 1, article no. 245, 2013.

[25] J. Henderson and R. Luca, "Positive solutions for a system of fractional differential equations with coupled integral boundary conditions," Applied Mathematics and Computation, vol. 249, pp. 182-197, 2014.

[26] J. Henderson, R. Luca, and A. Tudorache, "On a system of fractional differential equations with coupled integral boundary conditions," Fractional Calculus and Applied Analysis, vol. 18, no. 2, pp. 361-386, 2015.

[27] J. Jiang, L. Liu, and Y. Wu, "Symmetric positive solutions to singular system with multi-point coupled boundary conditions," Applied Mathematics and Computation, vol. 220, pp. 536-548, 2013.

[28] J. Jiang, L. Liu, and Y. Wu, "Positive solutions to singular fractional differential system with coupled boundary conditions," Communications in Nonlinear Science and Numerical Simulation, vol. 18, no. 11, pp. 3061-3074, 2013.

[29] C. Yuan, D. Jiang, D. O’Regan, and R. P. Agarwal, "Multiple positive solutions to systems of nonlinear semipositone fractional differential equations with coupled boundary conditions," Electronic Journal of Qualitative Theory of Differential Equations, no. 13, 17 pages, 2012.
[30] Y. Zou, L. Liu, and Y. Cui, "The existence of solutions for fourpoint coupled boundary value problems of fractional differential equations at resonance," Abstract and Applied Analysis, Article ID 314083, 8 pages, 2014.

[31] D. J. Guo and V. Lakshmikantham, Nonlinear Problems in Abstract Cones, vol. 5 of Notes and Reports in Mathematics in Science and Engineering, Academic Press, New York, NY, USA, 1988. 


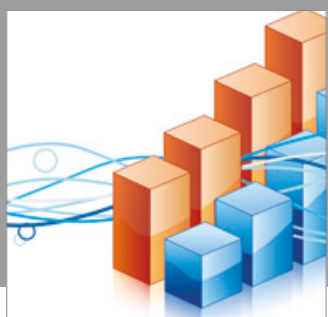

Advances in

Operations Research

vatersals

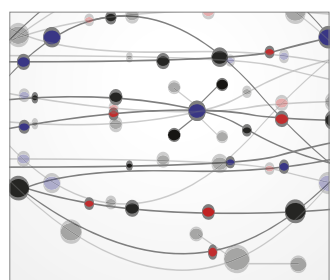

\section{The Scientific} World Journal
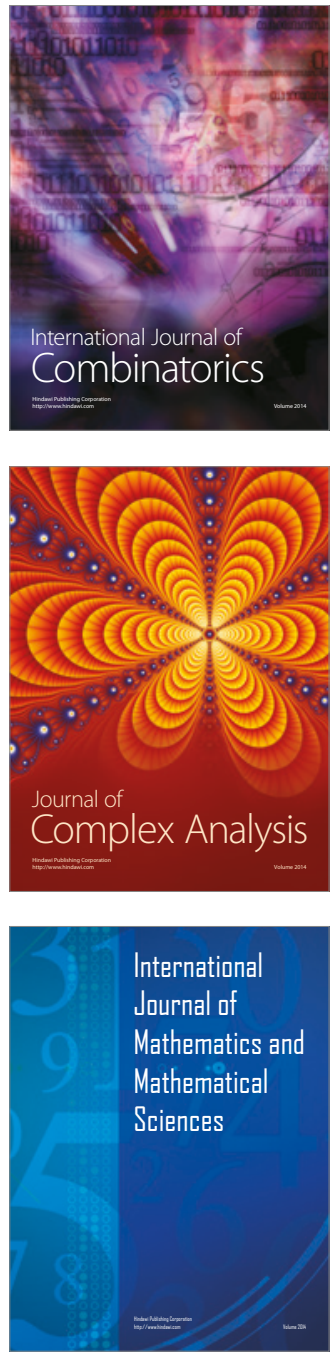


Algebra

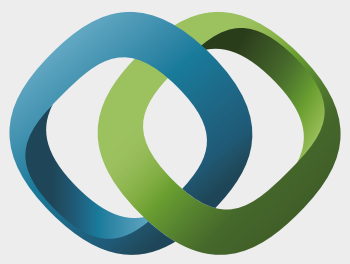

\section{Hindawi}

Submit your manuscripts at

https://www.hindawi.com
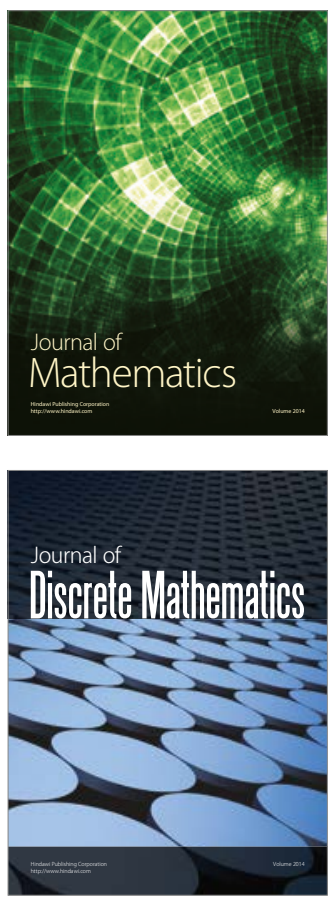

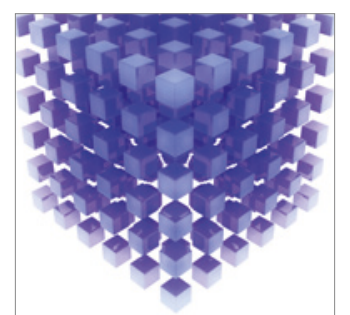

Mathematical Problems in Engineering
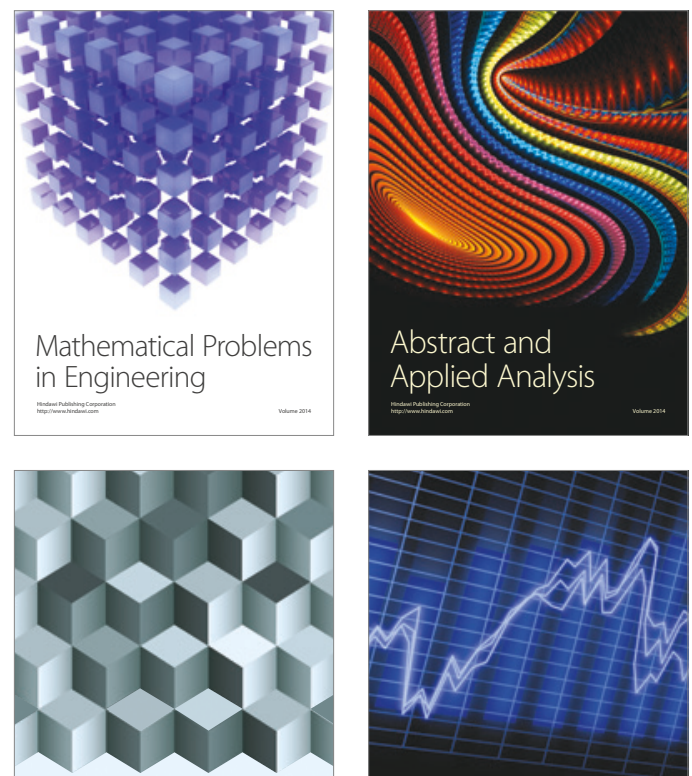

Journal of

Function Spaces

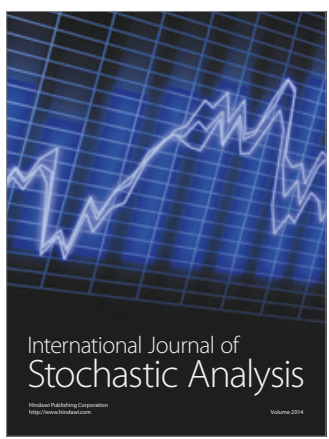

Probability and Statistics
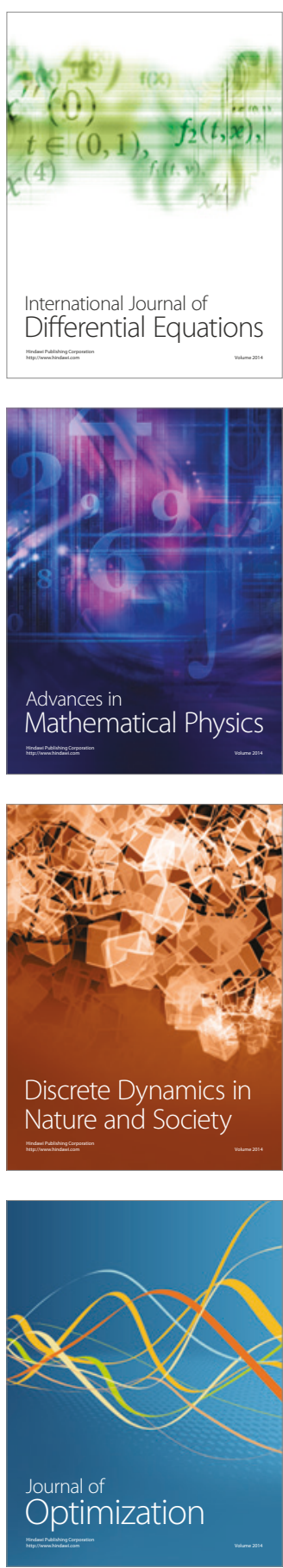